\title{
Nanoparticles-mediated genetic delivery of growth inhibiting genes on balloon angioplasty to suppress intimal hyperplasia
}

\begin{abstract}
In the U.S. alone, over 200,000 cardiovascular surgical procedures utilizing venous grafts fail annually, primarily due to restenosis caused by the physiological healing response known as intimal hyperplasia (IH). IH can be attributed to endothelial cell proliferation, narrowing the walls of the blood vessels through the role of key genes Pdx 1 and VEGF-A. Pdx1 is a home box whose promoter can induce over expression of VEGF-A, a gene which allows for the growth of the intima. While many genetic therapies work in vitro, targeted delivery in vivo often proves infeasible, rendering such therapies unusable. It was hypothesized that genes that could interfere with IH could be contained within Nanoparticle and coated onto a surgical balloon in order to specifically and efficiently target the region of interest. shRNA-regulated knock down of Pdx 1 delivered to a rat carotid artery via nanoparticle could lower the expression of VEGF-A, thereby preventing endothelial growth, the development of a neo-intima and ultimately occlusion. Scanning electron microscopy, transmission electron microscopy, fluorescence and confocal light microscopy, and realtime polymerase chain reaction were used in the analysis of samples to assess the extent of $\mathrm{IH}$ and levels of VEGF-A. In vitro analysis also showed evidence of fluorescence, confirming the efficacy of the nano-particle vector. Significant results $(\mathrm{P}<0.01)$ support the proposed hypothesis. While intimal hyperplasia was the model chosen for study in this experiment, further research hopes to apply the method to various venous and arterial conditions.
\end{abstract}

Keywords: hyperplasia, arteriotomy, stenosis, angioplasty, nanoparticle
Volume I Issue 2 - 2014

Serena Tharakan, Di Rito
Academy for Medical Science Technology, USA

Correspondence: Serena Tharakan,Academy for Medical Science Technology, USA, Tel 20I-937-4893, Email sertha@bergen.org

Received: April 14, 2014 | Published: May 09, 2014

\section{Introduction}

More than 500,000 coronary bypass surgeries are performed annually in the United States. ${ }^{1}$ Over $40 \%$ of these procedures fail, primarily due to restenosis, or the recurrence of an abnormal narrowing of an artery or valve after corrective surgery. ${ }^{2}$ Such arterial narrowing can block the flow of blood necessary for survival. Restenosis, the process of tissue regrowth into the vessel, occurs at a rate of $25 \%$ to $50 \%$ in balloon angioplasties-a surgical procedure in which a balloon catheter widens a blocked or narrowed blood vessel, most commonly an artery. ${ }^{3}$ The primary mechanism contributing to restenosis is the body's natural physiological healing response to vessel injury, known as intimal hyperplasia.

Intimal hyperplasia can be attributed to endothelial cell proliferation, narrowing the walls of arteries as the injured endothelium releases inflammatory mediators. This ultimately replicates scar formation with migration of smooth muscle cells from the media to the intima, creating what is known as a neo-intima, which then usually obstructs the artery. It has been observed that this response is accelerated when prosthetic material remains in the vessel. Such is the case with the placement of stents originally used to correct this matter. Stenosis derived from cases of intimal hyperplasia has proven difficult to treat as the stenoses are firm and require prolonged high inflation pressures to dilate with a balloon. The stenoses of the vessel often recur and repeated treatment with the use of balloon angioplasty only work to further perpetuate the healing response mechanism. ${ }^{4}$

In prior studies, the role the Vascular Endothelial Growth Factor A (VEGF-A) gene plays in intimal hyperplasia has been an area of great inquiry. VEGF-A is a gene that induces the development of blood vessels by stimulating endothelial cell growth. Because intimal hyperplasia is in essence the excess regrowth of the innermost endothelial layer, the intima, it is believed that VEGF-A is a factor associated with accelerating restenosis. ${ }^{5}$ Pancreatic and duodenal homeobox $1(\mathrm{Pdx} 1)$ is a separate gene that has generally been studied regarding its role in diabetes. ${ }^{6}$ The relationship between Pdx1 and VEGF-A has been poorly investigated, especially in regards to intimal hyperplasia. It has been suggested that Pdx1 may be capable of inducing over expression of VEGF-A. Because over expression of VEGF-A is associated with increased levels of intimal hyperplasia, silencing VEGF-A via Pdx1 can theoretically reduce such intima thickening. It was hypothesized that if $\mathrm{Pdx} 1$ were silenced using a system called RNA interference, the expression of VEGF-A would decline, thereby preventing excessive endothelial cell re-growth and therefore neo-intima formation. This would also provide further information about the relationship between Pdx1, VEGF-A, and intimal hyperplasia, which is currently inadequately documented in the scientific literature.

Another problem scientist currently faces lies in targeted delivery of genetic therapies. Naked DNA is unstable in the blood, and will rapidly degrade. Retro and lenti-viral vectors have the potential for oncogenicity and insertional mutagenesis of host cell genes due to random chromosomal integration. ${ }^{7}$ Cationic lipids for gene delivery also show a high level of toxicity and a low level of efficiency that renders them non-viable for clinical usage. Nanoparticles are an attractive option for gene therapy as they can be manipulated for targeted delivery. Furthermore, they have high storage stability, low 
manufacturing cost, low immunogenicity, and resistance to microbial attack. Unlike naked DNA, the nano-particles prevent degradation of nucleic acids as nano-sized materials of $100 \mathrm{~nm}$ have been shown to efficiently trap DNA or RNA and allow its escape from the endosome. ${ }^{8}$ Finally, a method commonly used for gene silencing is RNA interference via shRNA, which utilizes short hairpin RNA expressed from delivered plasmid DNA to impede mRNA signaling processes of specific genes. The DNA necessary for RNA interference can be packaged in plasmids within nano-particles. The anti-intimal hyperplasia genes encapsulated in nano-particles would ultimately break the aforementioned detrimental cycle of the healing and treatment process, saving money, time, and lives.

\section{Materials and methods}

\section{Plasmid preparation}

shRNA vectors against $\mathrm{Pdx} 1$ gene were obtained from Origene (Figure 1). The vectors contained an anti-Pdx1 sequence, designed to silence the Pdx1 gene, as well as a green fluorescent protein (GFP) sequence, designed to allow cells that take up the vector to glow green under fluorescent light, a factor which was critical during data analysis. The vector quantity was amplified using $E$. Coli and then via a Maxi-prep, as previously described ${ }^{9}$.

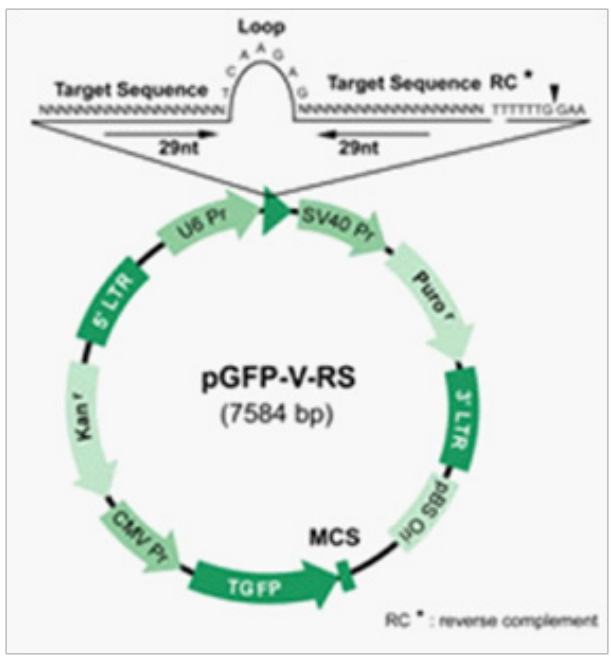

Figure I Gene Map for Anti-PdxI Plasmid . ${ }^{13}$

This figure outlines the gene map for the anti-PdxI plasmid. The target sequence in question is an anti-PdxI sequence.Another important component of the vector is the GFP sequence, which allows all cells that successfully take up the vector to glow green under UV light.

\section{Nanoparticle preparation}

PLGAnano-particles were then prepared according to a customized protocol. ${ }^{10}$ PLGA 50:50 (100mg) and Pdx1 shRNA plasmid (500 $\mu 1$ of $400 \mu / \mathrm{ml}$ ) were used in the preparation of the nano-particles. Plasmid in TE buffer $(500 \mu \mathrm{l})$ was poured into the organic phase and sonicated using a probe sonicator at $10 \mathrm{~W}$ for $1 \mathrm{~min}$ to form the primary emulsion. Following sonication, the emulsion was poured into $8 \mathrm{ml}$ of $2 \%$ PVA and sonicated at $30 \mathrm{~W}$ for $3 \mathrm{~min}$. After sonication, the double emulsion was poured into $50 \mathrm{ml}$ of $2 \%$ PVA, and solvent was allowed to evaporate at moderate stirring at $1500 \mathrm{rpm}$ in a magnetic stirrer overnight at room temperature. The suspension was further evaporated using a rotovap for $2 \mathrm{~h}$ at $40^{\circ} \mathrm{C}$ in a heated water bath to remove residual solvent. Following evaporation, the suspension was centrifuged at $10,200 \mathrm{rpm}$ for $50 \mathrm{~min}$ at $4^{\circ} \mathrm{C}$. The particle pellet was resuspended in double-distilled water $(20 \mathrm{ml}$; repeated twice to remove surface bound PVA and plasmid) and lyophilized. After preparation, nano-particles were analyzed under TEM for size and quality. Once the DNA-containing nano-particles were created, they were employed during animal surgeries.

\section{Pre-operative procedures}

All animal procedures were approved by the hospital and by Institutional Animal Care and Use Committees. Prior to animal contact, the surgeon put on a surgical mask, surgical gloves, hair net, surgical shoe coverings, and a mask to minimize risk of any possible infections. Before the surgery, the balloon catheter and trocar were prepared as previously described. ${ }^{11} \mathrm{~A}$ surgical plane of anesthesia was maintained for the duration of the surgery. This level of anesthesia was monitored throughout the surgery, and additional anesthesia was administered as necessary. The rat was placed in the induction chamber to begin anesthesia and isoflurane was introduced. After roughly 5 minutes, the rat was moved to a heating pad attached to a breathing tube, at which point the isoflurane was reduced. The rat was restrained with tape and the eyes lubricated with ophthalmic solution. Saline was also administered for hydration.

\section{Surgery proper}

The neck of the rat was shaved for exposure for surgery. Nair ${ }^{\circledR}$ was used to remove any remaining fur. This area was then swabbed alternately with betadine and absolute alcohol swabs. At this point, the surgical area was cleansed with $70 \%$ alcohol and set up underneath the microscope, with all instruments, suture, cotton-tipped applicators, tape, gauze, etc. properly sterilized and placed on top of the sterile field. A hole was cut in the sterile field to allow access to the rat's neck, at which point surgery began. Figures $2 \mathrm{a}-2 \mathrm{e}$ illustrate the detailed methodology of surgery proper.

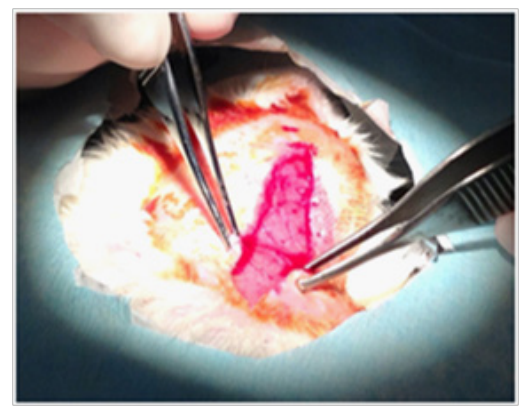

Figure 2a Surgical Procedure, This shows the incision made on the neck of the rat from just below the thyroid glands to the sternum.

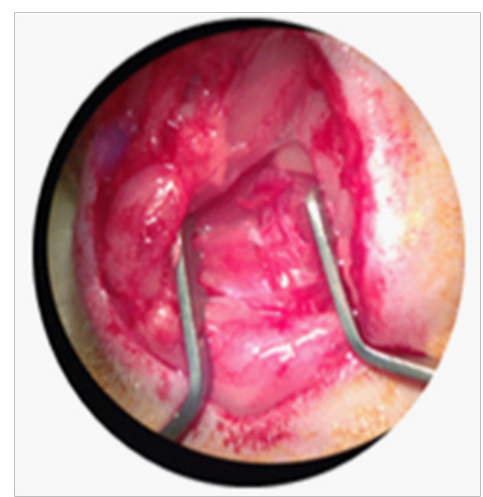

Figure 2b Surgical Procedure, After glandular tissue dissection, muscles were retracted to expose the carotid artery. 


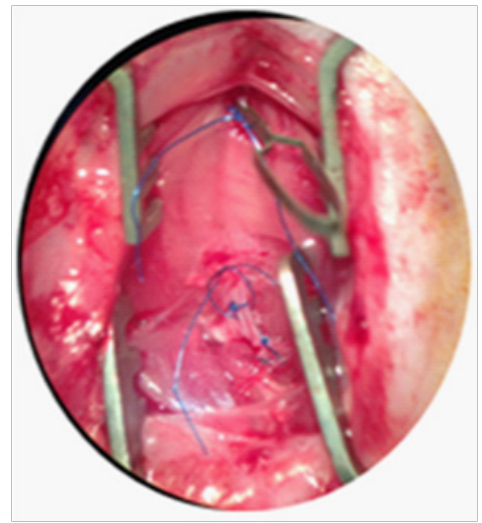

Figure 2c Surgical Procedure, Sutures were placed as previously described upon the external branch, internal branch, and common of the carotid artery. The suture on the common was supplemented with a surgical clamp to securely prevent blood flow to the carotid.

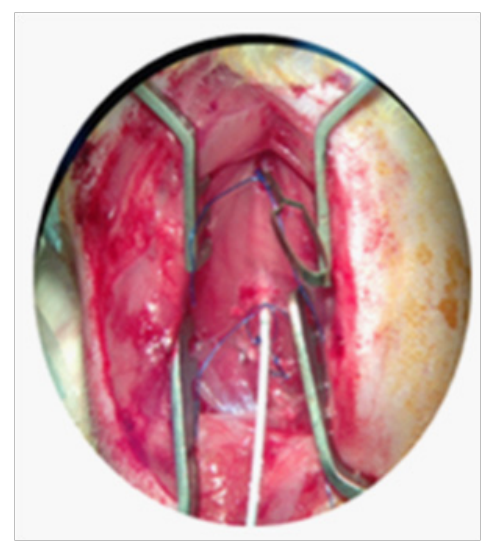

Figure 2d Surgical Procedure, Once all sutures had been placed, the balloon, which in the experimental condition was immersed into the glycerol-nanoparticle solution, was inserted into the lumen of the common carotid until the aortic arch, inflated, and retracted three times.

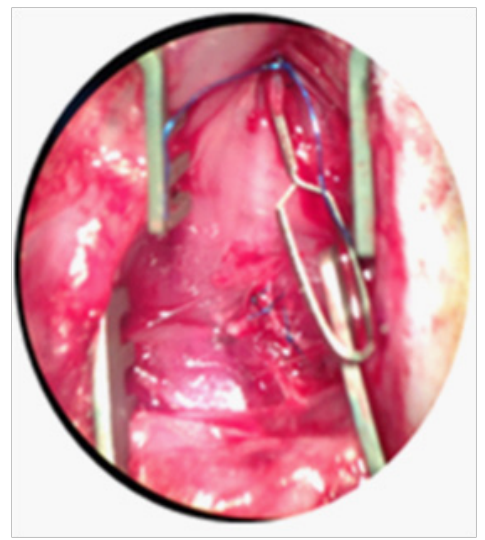

Figure 2e Surgical procedure.After the inflation procedure, the sutures were tied off and blood flow was reestablished.

First, the incision was made with a No. 10 blade scalpel, starting just below the chin of the animal towards the direction of the tail, stopping just above the ribcage at the sternum. Blunt forceps were used to carefully dissect the skin from the underlying glandular tissue. Next, muscle tissues were separated using $3 \mathrm{~S}$ and $5 \mathrm{~S}$ forceps and blunt dissection was performed along the longitudinal left aspect of the central and adjacent muscular tissues (sternocleidomastoid, omohyoid, thyrohyoid, sternohyoid). A retractor was utilized to draw back skin and muscular tissues in order to visualize the left carotid artery and vagus nerve. If arterial branching off the external carotid artery was apparent (Figure 2c), the ascending pharyngeal, occipital and/or superior thyroid arteries were completely tied off using short lengths of 6-0 Prolene suture. A 5-6" 6-0 Prolene suture was tied on the external artery branch as distally and far away from the bifurcation as possible. Distal to the bifurcation of the common carotid artery, a loosely tied 3" 6-0 Prolene blue monofilament suture was looped around the external carotid artery branch. The internal carotid artery was then tied off using a $\sim 3$ " 4-0 Prolene blue monofilament suture. At the most proximal site on the left common carotid artery, as close to the sternum as possible, single $\sim 3$ " suture, size $4-0$, Prolene, was placed around the artery. The area was then incubated with 1.5 ccs of lidocaine for several minutes. The left common carotid artery was clamped proximally near the loose suture in order to stop common carotid artery blood flow. The arteriotomy was then made between the two sutures on the external carotid using micro scissors. The trocar guiding needle was used to gently insert the un-inflated balloon into the arteriotomy hole. The balloon was then advanced all the way to the arterial clamp on the common carotid artery. The clamp was removed on the common carotid and the catheter was passed all the way down to the aortic arch. The balloon was then inflated and backed out almost all the way to the arteriotomy where it was then deflated. This process of inflation and deflation occurred three times per surgery. After the balloon was fully removed, the clamp was placed back on the proximal common carotid, and the loose suture on the external immediately distal to the arteriotomy was tied off. The clamp and the loose on the common were then removed. After blood flow was reestablished, the suture on the internal was removed as well. Overlying tissues were then replaced and glandular tissues were closed with a 6-0 Prolene running stitch. The skin was then closed with interrupted sutures using 6-0 Prolene.

\section{Post-operative procedures}

Once the animal was sutured, ketorolac, an analgesic, was immediately provided and anesthesia halted. The rat was cleaned using gauze and sterile water and then placed on a heating pad and monitored to ensure comfort. Once the rat had displayed signs of alertness and was ambulatory, it was returned to its cage and given sufficient food and drink. Instruments were then thoroughly cleaned and sterilized. The surgical area was also swabbed with $70 \%$ alcohol and disinfected. Finally, each animal was given daily assessments for two weeks following the surgery to ensure normal and healthy recovery.

\section{Surgery variables}

In total, nine surgeries were performed on nine separate rats. The initial two surgeries represented the first control group, standard left carotid artery injury surgeries, as previously described. The next two surgeries comprised the second control group, rats which received a standard left carotid artery injury surgery, modified slightly to include a glycerol coating without nano-particles on the catheter to test whether or not the substance had any noteworthy effects on the artery and/ or the overall health of the animal before the nano-particle treatment was delivered. The balloon was immersed in glycerol until $100 \mu \mathrm{L}$ had covered its surface. Another two surgeries served as an experimental group to compare the method of delivery with the glycerol-coated balloon to a more direct method of delivery: infusion. After the 
arteriotomy was performed, the artery was cannulated (filled) with a syringe of $100 \mu \mathrm{L}$ of $50 \%$ glycerol and flushed. The procedure then proceeded as previously described. The last three surgeries served as the experimental group utilizing the nano-particles suspended in the glycerol that coated the balloon.

After two weeks, each rat was euthanized via perfusion fixation. ${ }^{12}$ This allowed for tissue harvesting of the artery. The tissues were then sent to histology departments, which performed a tri-chrome stain. The tri-chrome stained slides were analyzed via Image-J, NIH software which determines the amount of intimal hyperplasia. Finally, the rest of the tissue harvested was dissolved in TRIZOL (Life Technologies), a solution capable of dissolving the artery into a stable form to preserve RNA for harvest. Once the RNA was harvested via a standard isolation protocol involving chloroform for phase separation, isopropyl alcohol for RNA precipitation, and ethanol for RNA wash, the RNA was made into cDNA via a Clontech cDNA synthesis kit. The kit uses reverse transcriptase to synthesis cDNA from the isolated RNA. cDNA was subsequently analyzed via rt-PCR. The rt-PCR runs the DNA through 40 cycles on a thermal cycler that rotates between three major steps: denaturation, annealing, and extension. During denaturation, the temperature is roughly $95^{\circ} \mathrm{C}$, which causes disruption of the DNA hydrogen bonds between complementary bases, yielding single stranded DNA. In annealing, the temperature is lowered to 50$65^{\circ} \mathrm{C}$, which allows the primers to anneal to the single stranded DNA template. DNA polymerase then binds to this primer-template hybrid and DNA to synthesize of the target sequence begins. Finally, during extension at $70-72^{\circ} \mathrm{C}$, the activity of the DNA polymerase is optimal and primer extension occurs at rates of up to 100 bases per second.

\section{Results}

Once the arteries were perfused from the rats they were either embedded in a non-stained paraffin or processed in a standard procedure for trichrome staining. This method clearly stains the three layers of the artery to define the intima, media, and adventitia. The stained arteries suggest that the intima of the experimental condition expressed no regrowth whereas the standard control arteries with no glycerol coating on the balloon, as expected, had significant neo-intima formation, $(p<0.01)$ as did the control with the glycerol coating $(\mathrm{p}<0.01)$. The intimal regrowth of the various conditions is visibly highlighted in (Figures 3-5). Intimal hyperplasia was quantified using a software program, Image $J$, developed by the National Institutes of Health, which calculates the intimal area through image analysis. The various intimal hyperplasia levels were graphed against the different conditions and are depicted in Figure 6. Rats that received gene delivery via infusion developed severe blood clotting which would eventually completely clog the artery. Thus, this avenue was not further explored as a method for inhibiting intimal hyperplasia, because it created even more serious issues. Next, gene delivery was confirmed using fluorescence microscopy. Because the packaged shRNA plasmids contained the gene for green fluorescent protein, if GFP gene was in fact delivered properly, the intimal cells would express the protein and therefore glow green. Under UV light, gene delivery was confirmed as the intimal cells of the experimental condition did, in fact, fluoresce. The highlights of these pictures are displayed in Figure 7.

Nanoparticles were analyzed under transmission electron microscopy for size and quality. The size was roughly between 100$200 \mathrm{~nm}$, the expected result. The spherical form and size of the nanoparticles (Figure 8) helped confirm that the preparation had been executed properly. Finally, reverse transcriptase polymerase chain reaction was performed on cDNA extracted from rat arteries. The rtPCR (Figure 9) was used to measure gene expression of particular genes, in particular VEGF-A. It was found that the experimental condition had 68x lower levels of VEGF-A than the glycerol-control, a significance of $6.29 \mathrm{e}-60$.

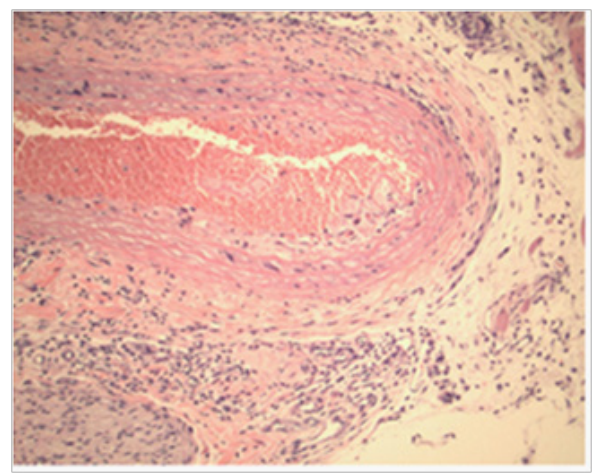

Figure 3 Standard Control Artery Cross-Section. This figure portrays a crosssection of a left carotid artery (100x) harvested from a rat that received a standard carotid artery injury serving as a control for the experiment. The highlighted portions of the vessel represent the areas of intimal regrowth, or intimal hyperplasia.

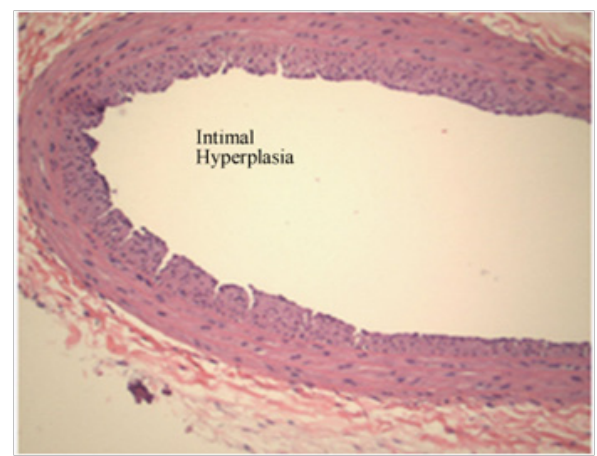

Figure 4 Glycerol Control Artery Cross-Section. This picture shows a crosssection of a left carotid artery (100x) harvested from a rat that was inflated with a glycerol-coated balloon, without the nano-particles containing the gene therapy. The highlighted portions of the vessel represent the areas of intimal regrowth, or intimal hyperplasia. This artery displays high levels of intimal hyperplasia, as would be expected.

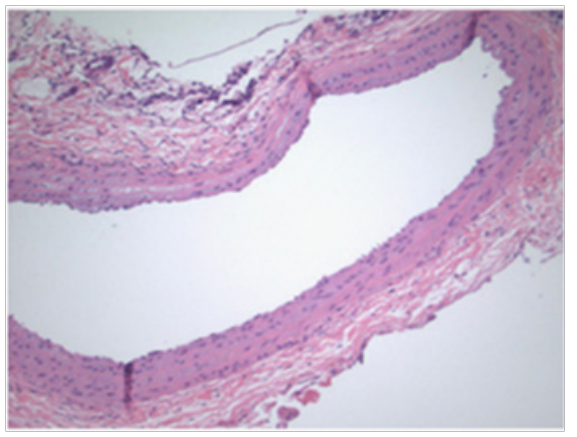

Figure 5 Experimental Artery Cross-Section. This picture shows a crosssection of a left carotid artery harvested from a rat that was inflated with the glycerol-nano-particle solution dipped balloon, which contained the gene therapy. The highlighted portions of the vessel represent the areas of intimal regrowth, or intimal hyperplasia. This artery displays very little presence of intimal hyperplasia, supporting the idea that the gene therapy was in fact delivered to the artery, thereby reducing the level of intimal hyperplasia. 


\section{Levels of Intimal Hyperplasia in Control vs. Experimental Rodents}

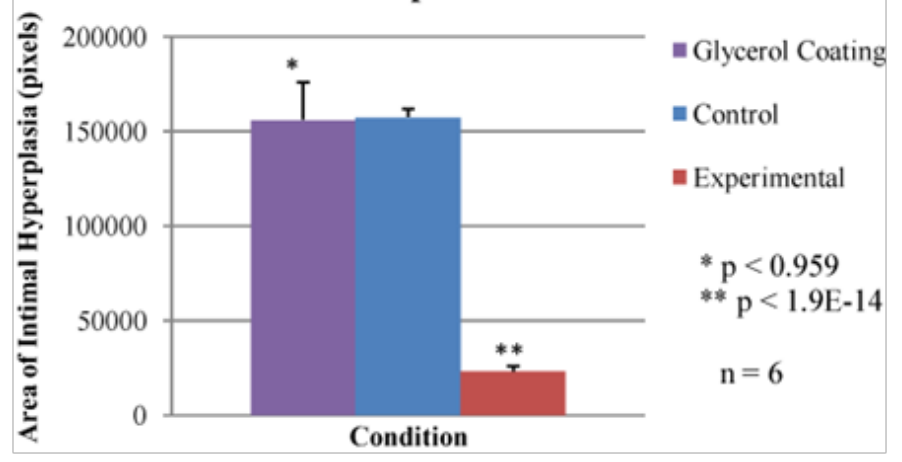

Figure 6 Levels of Intimal Hyperplasia. The figure shows the levels of intimal hyperplasia between the simple control (group I), the glycerol-only coated balloon control (group 2) and the glycerol-nano-particle experimental condition (group 3). It can be seen that while the difference between group and group 2 is insignificant ( $p$-value of 0.959 ), the difference between group 2 and group 3 is statistically significant ( $\mathrm{p}$-value of I.9e-14). This implies that glycerol alone has no significant effect on the levels of intimal hyperplasia, but glycerol containing nano-particles packaged with the genetic therapy did in fact lower the levels of intimal hyperplasia observed.

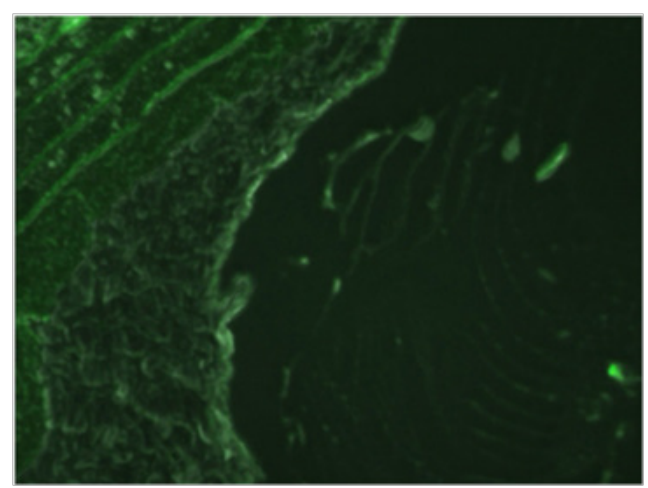

Figure 7 Fluorescence Microscopy of Experimental Artery. The above figure shows various photos displaying the fluorescence of the intimal layer under a UV light. Genetic delivery was further confirmed through fluorescence microscopy. Because the shRNA vectors contained the GFP gene, it was hypothesized that if nano-particle-mediated genetic delivery of the vectors was achieved, then the intimal cells would take up the GFP sequence, allowing them to glow green under fluorescent light.

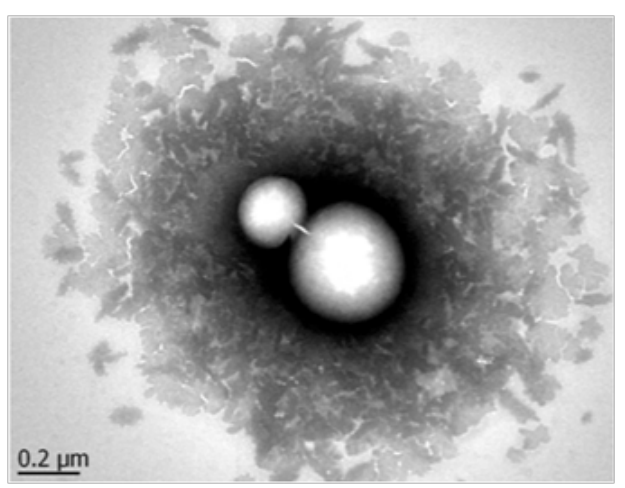

Figure 8 TEM Images of Nanoparticles. The figure shows a TEM image of PLGA nano-particles containing the anti-PdxI sequence.As can be seen from the scale, these nano-particles are roughly $200 \mathrm{~nm}$ in size and look properly formed, confirming the quality of the preparation.

\section{Relative Quantification of VEGF-A via rt-PCR}

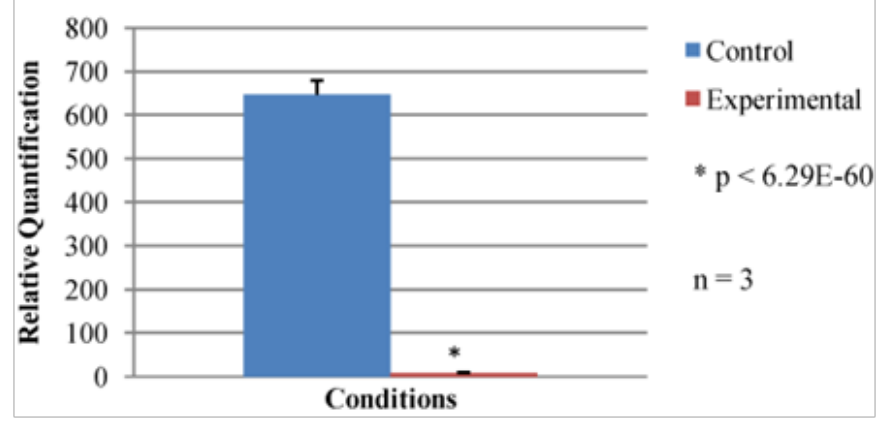

Figure 9 Relative Quantification of VEGF-A via rt-PCR. The figure shows the relative quantification of VEGF-A as determined by rt-PCR. The glycerolcontrol condition, seen in blue color, and the experimental condition, seen in red color, was compared to a standard, which was then used to determine relative quantification. It can be clearly seen that VEGF-A levels were significantly lowered $(6.29 \mathrm{e}-60)$ in the experimental condition as compared to the glycerol-control.

\section{Discussion}

The tri-chrome stain revealed that the experimental condition, rats receiving the nano-particle-mediated genetic therapy, suffered appreciably less intimal re-growth. This strongly suggests that these experimental rats did not develop intimal hyperplasia to the same degree that the control rats did. These data demonstrate that genetic delivery of anti-Pdx1 sequence to the vessel via nano-particles is a viable method of reducing the degree of intimal hyperplasia in vivo. This ensures the opening of the vessel for adequate blood flow following cardiovascular intervention.

The GFP data showed that the Pdx1 vector was indeed delivered to the vessel and expressed, as the Pdx1 vector contained the GFP gene. Because fluorescence was observed under UV light in experimental conditions, the genes must have been delivered. Furthermore, the fluorescence was observed in the exact location hypothesized. Normal rat arterial cells do not contain GFP gene, and thus a green fluorescence could only have resulted if these cells took up GFP gene from another source (the surgical delivery). Because the anti-Pdx 1 sequence and GFP gene were on the same plasmid, it can be assumed that the rats also took up the anti-Pdx1, which prevented intimal hyperplasia formation. More importantly, the fluorescence gives merit to the nanoparticle-mediated surgical delivery of genes. This idea is broader than intimal hyperplasia treatment. If nano-particles used in tandem with surgical procedures can deliver genetic therapies, this represents a novel, more efficient and more specific gene-delivery system for arterial and venous genes. The rt-PCR clarifies the relationship between Pdx1 and VEGF-A. When Pdx1 was knocked down via the treatment, VEGF-A levels were also significantly $(\mathrm{p}<0.01)$ reduced as confirmed by PCR. When VEGF-A levels were lowered, neo-intima materialization was precluded, as confirmed by the tri-chrome stain.

Another important finding was the impact of glycerol infusion. As previously discussed, pure glycerol infused as compared to pure glycerol coated on a balloon results in blood clot formation. This suggests that infusions of glycerol should not be used in arteries irrespective of the disease being treated. The only way this treatment differed from the pure glycerol coated on a balloon was in the method of distribution, further suggesting that while glycerol can be used for 
medical treatments in the body, the route of administration must be carefully chosen.

Significance of the findings presented stem from the elucidation of a new gene pathway. Pdx1 has previously shown indicative results that this gene could possibly play a role in the regulation of VEGF-A. This connection was furthered by evidence collected throughout the duration of this experimentation. With the controlled variable of the experimental surgeries being the absence or presence of Pdx 1 , and the ensuing analysis performed using rt-PCR; this experiment paved the pathway for the connection between these two genes and their roles in regulating intimal hyperplasia. This connection ultimately sets the stage for researchers to further investigate the role of Pdx1 in the multiple diseases and conditions associated with the VEGF-A gene. The development of an aggressive treatment for intimal hyperplasia will ultimately save a countless number of lives and drastically reduce patient expense and discomfort, as fewer surgeries will be needed once the cycle of re-stenosis is broken. In ending the necessity for repetitive detrimental balloon angioplasty and stent placement procedures, this method will save time, energy, and money. Without the need for multiple surgeries, patients have more sustainable and less susceptible immune systems, thus increasing the general health of those in need of the procedures.

The new method of gene delivery provides the ability to treat a multitude of both arterial and venous diseases. Theoretically the encapsulation of shRNA via nano-particles can be extended to any and all genetic diseases, and a surgical delivery can be employed in many cardiovascular surgeries. The applications of this treatment can be used and applied in a variety of ways, and while the model tested was specific to intimal hyperplasia, this protocol can be broadened to conditions such as coronary artery disease, the leading cause of death in the United States.

\section{Conclusion}

It was concluded that $\mathrm{Pdx} 1$ does in fact play a role in the suppression of intimal hyperplasia. Through silencing Pdx1 via gene therapy, lower levels of intimal hyperplasia were observed. Data also supported the idea that Pdx1 must regulate VEGF-A. Although it has been a loosely suggested idea prior in the literature, when Pdx 1 was knocked down, PCR confirmed that VEGF-A levels were also knocked down. Because VEGF-A is responsible for stimulating endothelial cell growth, with such low levels of this gene present in the artery, endothelial cell proliferation was inhibited. The data also supported a novel method of delivery for genetic therapies. Through encapsulation of shRNA plasmids in nano-particles subsequently coated on a surgical a balloon, genes were targeted and successfully delivered to cells in the carotid artery.

Another important conclusion reached was that the method of distribution of the glycerol affects the flow of blood in the artery. While glycerol coated on a balloon did not affect blood flow, the glycerol infusion resulted in a blood clot. It is possible that this is because with the balloon, the glycerol is more equally distributed throughout the artery, while the infusion concentrates the substance within the lumen, enabling obstruction of blood flow. In future research, this theory of blood flow and the mechanism of glycerol distribution will be tested. Any scientist performing studies that employ glycerol in the blood could hopefully use such data.

Alternatively, to refine the results of the experiment and investigate further avenues, a Gene Chip, a microchip which holds DNA probes that form half of the DNA double helix and can recognize DNA from samples being tested, can be tested for a variety of proteins associated with intimal hyperplasia. In addition to VEGF-A, Interleukin-6 (IL6), Mitogen-activated protein kinase (MAPK), guanosine nucleotidebinding proteins (G-Proteins) and other growth regulating proteins levels can be monitored. It would also be crucial to test gene expression levels in other tissues throughout the body to see whether the nano-particles deliver their genetic contents to solely the walls of the artery of interest or to other arteries in the area as well. Lack of intimal hyperplasia could have been attributed to the glycerol acting as a lubricating agent for the balloon and not contributing to arterial damage though this was shown to be highly unlikely through the extreme insignificance when the control surgeries were compared to the surgeries with the glycerol coated balloon. There is little prior literature documenting the phenomenon and specific treatment investigated, making it difficult to compare and review our results. However, previous literature does show a correlation between levels of VEGF-A expression and intimal hyperplasia, therefore confirming our results.

If a stent were to be coated with the nano-particle-glycerol solution and placed arterially, this would ultimately work to prevent the recurring cycle of stenoses that usually accompanies foreign objects such as stents that remain in the artery after the surgical procedures. By simply changing the shRNA vector for the specific gene of interest, various genetic diseases could be genetically treated. As gene delivery has consistently been a problem for scientists, this could represent an avant-garde innovation that facilitates gene therapy for arterial and venous disease.

\section{Acknowledgements}

No.

\section{Conflicts of interest}

None of the authors have any declared conflicts of interest.

\section{References}

1. http://www.med.umich.edu/cardiac-surgery/patient/adult/adultcandt/ cabg.shtml

2. Spencer FC, Isom OW, Classman E, et al. The long-term influence of coronary bypass grafts on myocardial infarction and survival. Ann Surg. 1974;180(4):439-451.

3. Hamid H, Coltart J. 'Miracle stents' a future without restenosis. Mcgill J Med. 2007;10(2):105-111.

4. http://radiopaedia.org/articles/intimal-hyperplasia

5. Shiojima I, Walsh K. The Role of vascular endothelial growth factor in restenosis. Circulation. 2013.

6. http://www.genecards.org/cgi-bin/carddisp.pl?gene=PDX1

7. Ali M, Lemoine NR, Ring CJ. The use of DNA viruses as vectors for gene therapy. Gene Ther. 1994;1(6):367-384.

8. Wagner DE, Bhaduri SB.Progress and outlook of inorganic nanoparticles for delivery of nucleic acid sequences related to orthopedic pathologies: a review. Tissue Eng Part B Rev. 2012;18(1):1-14.

9. https://www.mcdb.ucla.edu/Research/Banerjee/protocols/maxiprep-Qiagen.pdf

10. Song K, Lee H, Choung I, et al. The effect of type of organic phase solvents on the particle size of poly(D,L-lactide-co-glycolide) nanoparticles. Colloids and Surfaces A: Physicochem Eng Aspects. 2006;276(1-3):162-167. 
11. Tulis DA. Rat carotid artery balloon injury model. Methods Mol Med. 2007;139:1-30.
12. http://www.abcam.com/index.html?pageconfig=resource\&rid=11466

13. http://www.origene.com/shRNA/vector_information.aspx\#pGFP-V-RS 